\title{
MONOTONE MAPPINGS IN TOPOLOGICAL LINEAR SPACES
}

\author{
SADAYUKI YAMAMURO \\ (received 21 August 1964)
}

Let $E$ be a topological linear space over the real number field. Throughout of this paper, we denote by $G$ an open subset of $E$, by $\partial G$ the boundary of $G$ and by $\bar{G}$ the closure of $G$. The totality of all circled open neighbourhoods of the zero element is denoted by $\mathfrak{u}$.

The purpose of this paper is to introduce the notion of "monotone mappings" and to discuss their properties.

\section{Monotone mappings and their topological properties}

$A$ mapping $f: \bar{G} \rightarrow E$ is said to be increasing at $a \in G$ if there exists $U=U(t, a) \in \mathfrak{U}$ such that

(i) $a+U \subset G$.

(ii) It there exists $x \in \bar{U}$ such that

$$
f(a+x)-f(a)=\alpha x \text { and } x \neq 0,
$$

then $\alpha>0$.

If the condition (*) implies $\alpha \geqq 0$ or $\alpha<0$ or $\alpha \leqq 0$, the mapping is said to be non-decreasing or decreasing or non-increasing at a respectively.

It is clear that, if a mapping $f$ is decreasing or non-increasing at $a$, then $-f$ is increasing or non-decreasing at $a$. Therefore, in the sequel, we shall only deal with the increasing or non-decreasing mappings.

It is also clear that $f$ is increasing (or non-decreasing) at $a \in G$ if and only if the mapping

$$
f_{a}(x)=f(a+x)-f(a): \overline{U(f, a)} \rightarrow E
$$

is increasing (or non-decreasing) at 0 (the zero element of $E$ ).

Next, let us assume that the space $E$ is locally convex. A mapping $f: \bar{G} \rightarrow E$ is said to be a completely continuous vector field if $f$ is continuous and the image $F(\widetilde{G})$, where $F(x)=x-f(x)$, is contained in a compact subset.

Two completely continuous vector fields $f$ and $g$ are said to be strongly 
homotopic on $\bar{G}$ if there exists a mapping $h(x, t): \bar{G} \times[0,1] \rightarrow E$ which satisfies the following conditions:

$\left(\mathrm{H}_{1}\right) . h(x, 0)=f(x)$ and $h(x, 1)=g(x)$ for every $x \in \bar{G}$.

$\left(\mathrm{H}_{2}\right) . h(x, t) \neq 0$ for any $x \in \partial G$ and any $t \in[0,1]$.

$\left(\mathrm{H}_{3}\right)$. The set $\{x-h(x, t) \mid x \in \bar{G}, t \in[0,1]\}$ is contained in a compact set.

It is well-known that, if the completely continuous vector fields $f$ and $g$ are strongly homotopic on $\bar{G}$, then $d(0, G, f)=d(0, G, g)$, where $d(0, G, f)$ denotes the mapping degree of $G$ at 0 by $f$, provided that the both degrees are well defined (Leray-Schauder [1] or Nagumo [2]).

Now, we can prove that

THEOREM 1. Let E be locally convex. A completely continuous vector field $f: \bar{G} \rightarrow E$ is increasing at $a \in G$ if and only if the mapping $f_{a}$ is strongly homotopic to the identity mapping $I$ by $h(x, t)=(1-t) x+t f_{a}(x)$ on every such $\vec{V}$ that $V \in \mathfrak{U}$ and $V \subset U(f, a)$.

Proof. Let us assume that $f$ is increasing at $a$. It is clear that $f_{a}$ is also a completely continuous vector field and that $\left(\mathrm{H}_{1}\right)$ and $\left(\mathrm{H}_{3}\right)$ are satisfied for $f_{a}$ and $I$. Assume that there exists $V \in \mathfrak{U}$ such that $V \subset U(f, a)$ and $h\left(x_{0}, t_{0}\right)=0$ for some $x_{0} \in \partial V$ and for some $t_{0} \in[0,1]$. Since $x_{0} \neq 0$, we have that $t_{0} \neq 0$. Therefore,

$$
f_{a}\left(x_{0}\right)=\left(1-\frac{1}{t_{0}}\right) x_{0}
$$

which contradicts the assumption that $f_{a}$ is increasing at 0 . Conversely, let us assume that the mapping $h(x, t)=(1-t) x+t f_{a}(x)$ satisfies the conditions $\left(\mathrm{H}_{1}\right),\left(\mathrm{H}_{2}\right)$ and $\left(\mathrm{H}_{3}\right)$ on every such $\vec{V}$ that $V \in \mathfrak{U}$ and $V \subset U(f, a)$. If there exists a non-zero element $x_{0} \in \overline{U(f, a)}$ such that $f_{a}\left(x_{0}\right)=\alpha x_{0}$, we can find $V \in \mathfrak{U}$ such that $x_{0} \in \partial V$ and $V \subset U(t, a)$. Assume that $\alpha$ is not positive, then $t_{0}=1 /(1-\alpha)$ satisfies $0 \leqq t_{0} \leqq 1$, and, for this $t_{0}$, we have $h\left(x_{0}, t_{0}\right)=0$. This contradicts the condition $\left(\mathrm{H}_{2}\right)$. Thus, the proof is completed.

Theorem 1 immediately implies

THEOREM 2. Let $E$ be locally convex and a completely continuous vector field $f$ on $\bar{G}$ be increasing at $a \in G$. Take any $V \in \mathfrak{U}$ such that $V \subset U(f, a)$. Then, $d\left(0, V, f_{a}\right)=1$.

Next, we prove that an increasing completely continuous vector field on a locally convex $E$ is an open mapping. Note that, even if a mapping is increasing at every point of an open set, it is not necessarily one-to-one.

THEOREM 3. Let $E$ be locally convex. If a completely continuous vector field $f: \bar{G} \rightarrow E$ is increasing at every point of an open set $G_{1} \subset G$, then $f\left(G_{1}\right)$ is open. 
Proof. Let $b=f(a)$ and $a \in G_{1}$. We shall prove that $b$ is an interior point of $f\left(G_{1}\right)$. We can assume that $a+U(f, a) \subset G_{1}$. Since $f_{a}$ is increasing at 0 , for any non-zero $x \in \overline{U(f, a)}$, we have $f_{a}(x) \neq 0$, because, if $f_{a}(x)=0$ for some non-zero $x \in \overline{U(f, a)}$, we have $f_{a}(x)=0 \cdot x$ which contradicts the increasingness of $f$ at 0 . Therefore, $0 \notin f_{a}(\partial U)$ for $U=U(f, a)$, and since $f_{a}(\partial U)$ is closed, there exists $V \in \mathfrak{U}$ such that

$$
V \cap f_{a}(\partial U)=\phi \text {. }
$$

Take an element $y \in b+V$, and consider the completely continuous vector field

$$
g(x)=f(a+x)-y
$$

on $\bar{U}$. It is clear that $g(x) \neq 0$ for every $x \in \partial U$. Since

$$
f_{a}(x)-g(x)=y-f(a)=y-b \in V,
$$

we get, by Theorem 2 , that

$$
d(0, U, g)=d\left(0, U, f_{a}\right)=1 .
$$

Therefore, there exists $x \in U$ such that $g(x)=0$, that is, $y=f(a+x)$. Since $a+x \in a+U \subset G_{1}$, we have $y \in f\left(G_{1}\right)$. This completes the proof.

Remark. Our definition allows the existence of such a mapping $f$ that is increasing and decreasing at one point. For example, a mapping which has no small proper vectors is increasing and decreasing at 0 . But the argument above shows that, in case of odd-dimensional Euclidean space, any increasing mapping at one point cannot be decreasing at the same point. This follows from the fact that the decreasing mapping at 0 is strongly homotopic to $-I$ on the set $\bar{U}$ for some $U \in \mathfrak{U}$ and that $d(0, U,-I)=-1$ when the dimension is odd.

\section{Contraction vector fields and uniform monotony}

In this section, the space $E$ is assumed to be a real normed linear space.

A mapping $F: E \rightarrow E$ is said to be a contraction mapping if there exists a number $\gamma$ such that $0<\gamma<1$ and

$$
\|F(x)-F(y)\| \leqq \gamma\|x-y\| \text { for every } x \text { and } y \text { in } E .
$$

A mapping $f(x)=x-F(x)$, when $F(x)$ is a contraction mapping, is called a contraction vector field.

Also, we shall say that a mapping $f: \bar{G} \rightarrow E$ is unitormly increasing at $a \in G$ if there exist $U=U(f, a) \in \mathfrak{U}$ and $\varepsilon=\varepsilon(f, a)>0$ such that the following conditions are satisfied: 
(i) $a+U \subset G$.

(ii) If there exists a non-zero $x \in \bar{U}$ such that

then $\alpha>0$.

$$
\left\|f_{a}(x)-\alpha x\right\|<\varepsilon\|x\|,
$$

Obviously, if $f$ is uniformly increasing at $a$, then it is increasing at $a$. Then, we prove that

THEOREM 4. A contraction vector field $f$ is uniformly increasing at every point $a \in E$ and the set $U(f, a)$ can be taken to be the whole space $E$ for every $a \in E$.

Proof. Let $f(x)=x-F(x)$ and, for some $\gamma$ such that $0<\gamma<1$,

$$
\|F(x)-F(y)\| \leqq \gamma\|x-y\|
$$

Assume that there exists $x \in E$ such that $x \neq 0$ and

$$
\left\|f_{a}(x)-\alpha x\right\|<\varepsilon\|x\|,
$$

where $\varepsilon$ is an arbitrary number such that $\gamma+\varepsilon<1$. Then, for $F_{a}(x)=F(a+x)-F(a)$, we have

$$
\begin{aligned}
\varepsilon|| x \| & \geqq\left\|x-F_{a}(x)-\alpha x\right\|=\left\|(1-\alpha) x-F_{a}(x)\right\| \\
& \geqq|1-\alpha| \cdot|| x||-\left\|F_{a}(x)\right\| \geqq|1-\alpha| \cdot\|x\|-\gamma\|x\| .
\end{aligned}
$$

Since $\|x\| \neq 0$, we have $|1-\alpha| \leqq \varepsilon+\gamma<1$, which implies that $\alpha>0$.

Here is a possibility of generalizing the so-called "contraction mapping principle", namely, for an increasing mapping $f$ with some other properties, the equation $f(x)=0$ may have one and only one solution. We could not get the result which could be regarded as general enough. Here, as a partial answer, we prove the following

\section{THEOREM 5. Let us assume that}

(1). The mapping $f: E \rightarrow E$ is unitormly increasing at every $a \in E$ and $U(f, a)=E$ for every $a \in E$.

(2). There exists an element $a \in E$ such that $\|f(a)\|<\varepsilon(f, a) \cdot\|a\|$.

(3). For any $r>0$, the image of the set $S(a, r)=\{x \in E \mid\|a-x\| \leqq r\}$ by the mapping $F(x)$, where $F(x)=x-f(x)$, is contained in a compact subset.

Then, the equation $F(x)=x$ has one and only one solution in $S(a, r)$.

Proof. The condition $U(f, a)=E(a \in E)$ implies the unicity of the solution. In fact, if $F(x)=x$ and $F(y)=y$, since $f$ is increasing at $y$, we have

$$
0=f(x)-f(y)=f_{y}(x-y)=0 \cdot(x-y),
$$

which, by the definition of the increasingness, is impossible. The existence 
is shown as follows. For the element $a$ in the condition (2), choose the set $S(a, r)$ such that 0 is an interior point of $S(a, r)$. The mapping

$$
g(x)=f(a+x)
$$

is a completely continuous vector field on the set $S(r)=\{x \in E \mid\|x\| \leqq r\}$. We prove that $g(x)$ is strongly homotopic to the identity mapping on $S(r)$ by

$$
h(x, t)=(1-t) x+\operatorname{tg}(x) .
$$

We have only to show that $\left(\mathrm{H}_{2}\right)$ is true. Let us assume that $h\left(x_{0}, t_{0}\right)=0$ for some $x_{0} \in \partial S(r)$ and some $t_{0} \in[0,1]$. Then, since $t_{0} \neq 0$, we have

$$
\begin{aligned}
\left\|f_{a}\left(x_{0}\right)-\left(1-\frac{1}{t_{0}}\right) x_{0}\right\| & =\left\|\left[f\left(a+x_{0}\right)-\left(1-\frac{1}{t_{0}}\right) x_{0}\right]-f(a)\right\| \\
& =\left\|\frac{1}{t_{0}}\left[t_{0} g\left(x_{0}\right)+\left(1-t_{0}\right) x_{0}\right]-f(a)\right\| \\
& =\|f(a)\|<\varepsilon(f, a) \cdot\|a\|<\varepsilon(f, a) \cdot r \\
& =\varepsilon(f, a) \cdot\|x\|,
\end{aligned}
$$

hence it follows that $1-1 / t_{0}>0$, which is impossible. Therefore, there exists an element $x_{0} \in S(r)$ such that $0=g\left(x_{0}\right)=f\left(a+x_{0}\right)$, namely, $F\left(a+x_{0}\right)=a+x_{0}$.

We need the following fact later.

Theorem 6. Let a mapping $f: \bar{G} \rightarrow E$ be uniformly non-decreasing at $a \in G$, then, for any number $\alpha$ such that

$$
\left\|f_{a}(x)-\alpha x\right\|<\frac{1}{2} \varepsilon(f, a) \cdot\|x\| \text { for some non-zero } x \in \overline{U(f, a)},
$$

we have $\alpha \geqq \frac{1}{2} \varepsilon(f, a)$.

Proof. Put $\varepsilon_{0}=\varepsilon(f, a)$, then, if

$$
\left\|f_{a}(x)-\alpha x\right\|<\frac{1}{2} \varepsilon_{0}\|x\|, x \neq 0 \text { and } x \in \overline{U(f, a)} \text {, }
$$

we have

$$
\begin{aligned}
\left\|f_{a}(x)-\left(\alpha-\frac{1}{2} \varepsilon_{0}\right) x\right\| & =\left\|\left[f_{a}(x)-\alpha x\right]+\frac{1}{2} \varepsilon_{0} x\right\| \\
& \leqq\left\|f_{a}(x)-\alpha x\right\|+\frac{1}{2} \varepsilon_{0}\|x\|<\varepsilon_{0}\|x\|,
\end{aligned}
$$

hence it follows that $\alpha \geqq \frac{1}{2} \varepsilon_{0}$.

This theorem shows that the uniform non-decreasingness at a point coincides with the uniform increasingness at the same point. Therefore, in the sequal, we shall only deal with the uniform increasingness.

Finally, we have to give a remark on the uniform increasingness. Let $f: \bar{G} \rightarrow E$ be uniformly increasing at $a \in G$. Then, there exist $\varepsilon(f, a)$ and 
$U(f, a)$ such that $a+U(f, a) \subset G$ and " $\left\|f_{a}(x)-\alpha x\right\|<\varepsilon(f, a) \cdot\|x\|$ for some non-zero $x \in \overline{U(f, a)}$ " implies $\alpha>0$. This is equivalent to the following proposition: "If $\alpha \leqq 0$, then $\left\|f_{a}(x)-\alpha x\right\| \geqq \varepsilon(f, a) \cdot\|x\|$ for every non-zero $x \in \overline{U(f, a)}$."

Therefore, putting $\alpha=0$,

$$
\left\|f_{a}(x)\right\| \geqq \varepsilon(f, a) \cdot\|x\| \text { for every } x \in \overline{U(f, a)} .
$$

In the case of the continuous linear operators, this means that the operator has the continuous inverse. Therefore, unless the space $E$ is finite-dimensional, any completely continuous linear operator cannot be uniformly increasing. However, since every contraction vector field is uniformly increasing at every point, every completely continuous linear vector field is uniformly increasing if the norm of the completely continuous part is less than one.

This fact indicates that the study of uniform increasingness should mainly be concerned with the vector fields, rather than completely continuous mappings.

\section{Fréchet-derivatives}

In this section, the space $E$ is assumed to be a normed linear space over the real number field.

Our definition of the non-decreasingness at a point $a \in E$ of a mapping $f$ means that, if the mapping $f_{a}$ has a small proper element, the corresponding proper value must be non-negative. This fact immediately suggests the following

THEOREM 7. (1). If $f: \bar{G} \rightarrow E$ is non-decreasing at $a \in G$, then the bifurcation points of $f_{a}$, if such points exist, are non-negative. (2). If $f: \bar{G} \rightarrow E$ is uniformly increasing at $a \in G$, then the bifurcation points, if such points exist, are positive.

Proof. (1). Let $\lambda_{0}$ be a bifurcation point of $f_{a}$. Then there exist a. sequence $\left\{\lambda_{n}\right\}$ and a sequence $\left\{x_{n}\right\}$ such that

$$
\lim _{n \rightarrow \infty} \lambda_{n}=\lambda_{0}, \lim _{n \rightarrow \infty} x_{n}=0 \text { and } f_{a}\left(x_{n}\right)=\lambda_{n} x_{n} \quad(n=1,2, \cdots) .
$$

This implies, since $f_{a}$ is non-decreasing at 0 , that $\lambda_{n} \geqq 0$, and hence it follows that $\lambda_{0} \geqq 0$.

(2). For this sequence $\left\{\lambda_{n}\right\}$, we have, by Theorem 6 , that $\lambda_{n} \geqq \frac{1}{2} \cdot \varepsilon(f, a)$. Therefore, $\lambda_{0}$ is positive.

It is well known that, if $f_{a}$ is Fréchet-differentiable at 0 , then any bifurcation point of $f_{a}$ is a proper value of the Fréchet-derivative $d f_{a}(0, x)$ 
of $f_{a}(x)$ at 0 . This fact suggests that there is a close connection between the monotonicity of the mapping $f_{a}(x)$ and the sign of the proper values of $d f_{a}(0, x)$. In order to establish this connection exactly, we need a new definition.

A mapping $f: \vec{G} \rightarrow E$ is said to be strongly increasing (or strongly nondecreasing) at $a \in G$ if the existence of a sequence $\left\{x_{n}\right\}$ such that

$$
x_{n} \neq 0, \lim _{n \rightarrow \infty} x_{n}=0 \text { and } \lim _{n \rightarrow \infty} \frac{\left\|f_{a}\left(x_{n}\right)-\alpha x_{n}\right\|}{\left\|x_{n}\right\|}=0
$$

implies $\alpha>0$ (or $\alpha \geqq 0$ ).

It is clear that, if a mapping $f$ is uniformly increasing at $a$, then $f$ is strongly increasing at $a$. The converse may not always be true. In the paper [3], we have given the following definition: A mapping $f: \bar{G} \rightarrow E$, where $0 \in G$, is said to be linearly lower bounded, if there exist $\gamma>0$ and $\delta>0$ such that $S(\delta)=\{x \in E \mid\|x\| \leqq \delta\} \subset \bar{G}$ and

$$
\|f(x)\| \leqq \gamma \cdot\|x\| \text { for every } x \in S(\delta) .
$$

Making use of this terminology, we can prove that

THEOREM 8. If a mapping $f: \bar{G} \rightarrow E$ is strongly increasing at $a \in G$ and the mapping $f_{a}$ is linearly lower bounded, then $f$ is uniformly increasing at $a$.

Proof. Let us assume that $f$ is not uniformly increasing at $a$. Then, we can find $\left\{x_{n}\right\}$ and $\left\{\alpha_{n}\right\}$ such that

$$
\left\|f_{a}\left(x_{n}\right)-\alpha_{n} x_{n}\right\| \leqq\left\|x_{n}\right\| / n, 0<\left\|x_{n}\right\| \leqq 1 / n \text { and } \alpha_{n} \leqq 0 .
$$

Since $\left|x_{n}\right| \cdot\left\|x_{n}\right\| \leqq\left\|f_{a}\left(x_{n}\right)\right\|+(1 / n) \cdot\left\|x_{n}\right\|$ and $f_{a}$ is assumed to be linearly lower bounded, the sequence $\left\{\alpha_{n}\right\}$ is bounded. Therefore, a subsequence $\left\{\alpha_{n_{i}}\right\}$ can be chosen in such a manner that $\lim _{i \rightarrow \infty} \alpha_{n_{i}}=\alpha_{0}$ for some $\alpha_{0}$. Then,

$$
\varlimsup_{i \rightarrow \infty} \frac{\left\|f_{a}\left(x_{n_{i}}\right)-\alpha_{0} \cdot x_{n_{i}}\right\|}{\left\|x_{n_{i}}\right\|} \leqq \lim _{i \rightarrow \infty} \frac{\left\|f_{a}\left(x_{n_{i}}\right)-\alpha_{n_{i}} x_{n_{i}}\right\|}{\left\|x_{n_{i}}\right\|}+\lim _{i \rightarrow \infty}\left|\alpha_{n_{i}}-\alpha_{0}\right|=0 .
$$

Since $f$ is strongly increasing at $a$, the number $\alpha_{0}$ must be positive. But, this is impossible, because $\alpha_{n_{i}}$ are non-positive.

This theorem, by the same reason as in the case of the uniform increasingness, indicates that, when we make use of the strong increasingness property, we should mainly deal with the vector fields, not with especially the completely continuous mappings, because a completely continuous linear operator is linearly lower bounded.

Now, we prove that 
THEOREM 9. Let $f: \bar{G} \rightarrow E$ be a completely continuous vector field, so that the set $F(\bar{G})$, where $F(x)=x-f(x)$, is contained in a compact set. Let $u s$ assume that $f$ is Fréchet-differentiable at $a \in G$. Then, $f$ is strongly increasing (or strongly non-decreasing) at $a$ if and only if every proper value of the Fréchet-derivative $\mathrm{df}_{a}(0, x)$ is positive (or non-negative).

PROOF. Let us assume that $f$ is strongly increasing at $a$ and $\lambda_{0}$ is a proper value of $d f_{a}(0, x)$. Therefore, there exists $x_{0} \in E$ such that

$$
d f_{a}\left(0, x_{0}\right)=\lambda_{0} x_{0}
$$

Since the Fréchet-derivative $d f_{a}(0, x)$ is linear with respect to $x$,

$$
d f_{a}\left(0, t x_{0}\right)=\lambda_{0} t x_{0} \text { for every number } t,
$$

and, by the definition of the Fréchet-derivatives, we have

$$
\lim _{t \rightarrow 0} \frac{\left\|f_{a}\left(t x_{0}\right)-d f_{a}\left(0, t x_{0}\right)\right\|}{t}=0,
$$

or,

$$
\lim _{t \rightarrow 0} \frac{\left\|f_{a}\left(t x_{0}\right)-\lambda_{0} t x_{0}\right\|}{t}=0,
$$

from which, since $f_{a}$ is assumed to be strongly increasing at 0 , it follows that $\lambda_{0}>0$.

Conversely, let us assume that every proper value of $d f_{a}(0, x)$ is positive and there exists a sequence $\left\{x_{n}\right\}$ such that

$$
x_{n} \neq 0, \lim _{n \rightarrow \infty} x_{n}=0 \text { and } \lim _{n \rightarrow \infty} \frac{\left\|f_{a}\left(x_{n}\right)-\alpha x_{n}\right\|}{\left\|x_{n}\right\|}=0 .
$$

Here, we can assume that $\alpha \neq 1$. Then, since $f_{a}(x)=x-F_{a}(x)$, we have

$$
\lim \frac{\left\|(1-\alpha) x_{n}-F_{a}\left(x_{n}\right)\right\|}{\left\|x_{n}\right\|}=0,
$$

from which it follows that

$$
\lim _{n \rightarrow \infty} \frac{\left\|(1-\alpha) x_{n}-d F_{a}\left(0, x_{n}\right)\right\|}{\left\|x_{n}\right\|}=0,
$$

where $d F_{a}(0, x)$ is the Fréchet-derivative of $F_{a}(x)$ at 0 , the existence of which follows immediately from the Fréchet-differentiability of $f_{a}(x)$ at 0 . Since $d F_{a}(0, x)$ is linear with respect to $x$, we have, putting $y_{n}=x_{n} /\left\|x_{n}\right\|$, that

$$
\lim _{n \rightarrow \infty}\left\|(1-\alpha) y_{n}-d F_{a}\left(0, y_{n}\right)\right\|=0 .
$$

Since $d F_{a}(0, x)$ is a compact linear operator, there exists a subsequence $\left\{y_{n_{i}}\right\}$ and $y_{0} \in E$ such that 
and then we have

$$
\lim _{i \rightarrow \infty} d F_{a}\left(0, y_{n_{i}}\right)=y_{0}
$$

$$
\lim _{i \rightarrow \infty}(1-\alpha) y_{n_{i}}=y_{0} .
$$

Therefore, $\left\|y_{0}\right\|=|1-\alpha| \neq 0$, and

$$
y_{0}-d f_{a}\left(0, y_{0}\right)=d F_{a}\left(0, y_{0}\right)=(1-\alpha) y_{0},
$$

so that $\alpha$ is a proper value of $d f_{a}(0, x)$, hence it follows that $\alpha>0$.

\section{Perturbation}

Let $E$ be a normed linear space over the real number field. We begin with

THEOREM 10. Let $f: \bar{G} \rightarrow E$ be uniformly increasing at $a \in G$. Then, for any mapping $g: \bar{G} \rightarrow E$ such that $g_{a}(x)$ is linearly lower bounded, there exists $\varepsilon_{0}>0$ such that every $f(x)+\varepsilon g(x)\left(|\varepsilon| \leqq \varepsilon_{0}\right)$ is uniformly increasing at $a$.

Proof. Since $f$ is uniformly increasing at $a$, there exist $\varepsilon_{1}>0$ and $\delta_{1}>0$ such that $a+S\left(\delta_{1}\right) \subset \bar{G}$ and the existence of such non-zero $x \in S\left(\delta_{1}\right)$ that

$$
\left\|f_{a}(x)-\alpha x\right\|<\varepsilon_{1}\|x\|
$$

implies $\alpha>0$. Since $g_{a}$ is linearly lower bounded, there exists $\varepsilon_{2}>0$ and $\delta_{2}>0$ such that $a+S\left(\delta_{2}\right) \subset \widetilde{G}$ and

$$
\left\|g_{a}(x)\right\| \leqq \varepsilon_{2}\|x\| \quad \text { if } \quad\|x\| \leqq \delta_{2} .
$$

Put $\varepsilon_{0}=\varepsilon_{1} /\left(1+\varepsilon_{2}\right)$ and $\delta_{0}=\min \left\{\delta_{1}, \delta_{2}\right\}$, and assume that

$$
\left\|f_{a}(x)+\varepsilon g_{a}(x)-\alpha x\right\|<\varepsilon_{0}\|x\|,|\varepsilon| \leqq \varepsilon_{0} \text { and } 0<\|x\| \leqq \delta_{0} .
$$

Then,

$$
\begin{aligned}
\left\|f_{a}(x)-\alpha x\right\| & \leqq\left\|f_{a}(x)+\varepsilon g_{a}(x)-\alpha x\right\|+|\varepsilon| \cdot\left\|g_{a}(x)\right\| \\
& \leqq \varepsilon_{0}\left(\|x\|+\left\|g_{a}(x)\right\|\right) \leqq \varepsilon_{0}\left(1+\varepsilon_{2}\right)\|x\|=\varepsilon_{1}\|x\|,
\end{aligned}
$$

from which it follows that $\alpha>0$, namely, $f+\varepsilon g$ is uniformly increasing at $a$.

Next, we prove a perturbation theorem. For the proof, we use a fixed point theorem which has been proved on [4]. It is as follows:

$L$ et $E$ be a locally convex topological linear space over the real number field, and $G$ be an open set. Let $F$ be a completely continuous mapping of $\bar{G}$ in $E$. If there exists a point $a \in G$ such that

"if $F(x)=\alpha x+(1-\alpha)$ a for some $x \in \partial G$, then $\alpha \leqq 1 "$, then $F$ has at least one fixed point in $\bar{G}$.

Now, we prove the following 
THEOREM 11. Let $F$ be a completely continuous mapping of $\bar{G}$ and $a \in G$ be a fixed point of $F$. If the completely continuous vector field $f(x)=x-F(x)$ is uniformly increasing at a, then, for any completely continuous mapping $H$ of $\bar{G}$ such that $\left\|H_{a}(x)\right\|=o(\|x\|) \quad(\|x\| \rightarrow 0)$, there exists $\varepsilon_{0}>0$ such that every $F(x)+\varepsilon H(x)\left(|\varepsilon| \leqq \varepsilon_{0}\right)$ has at least one fixed point in some neighbourhood of $a$.

Proof. By the previous theorem, since $H_{a}(x)$ is linearly lower bounded, there exists $\varepsilon_{1}>0$ such that every $f(x)+\varepsilon H(x) \quad\left(|\varepsilon| \leqq \varepsilon_{1}\right)$ is uniformly increasing at $a$. Therefore, there exist $\varepsilon_{2}>0$ and $\delta_{1}>0$ such that $a+S\left(\delta_{1}\right) \subset \bar{G}$ and the existence of non-zero $x \in S\left(\delta_{1}\right)$ for which

$$
\left\|f_{a}(x)+\varepsilon_{1} H_{a}(x)-\alpha x\right\|<\varepsilon_{2}\|x\|
$$

implies $\alpha>0$. Next, choose $\varepsilon_{3}>0$ for which $\varepsilon_{1} \varepsilon_{3}<\frac{1}{2} \varepsilon_{2}$ and take $\delta_{0}$ such that

and

$$
\left\|x_{0}-a\right\| \leqq \delta_{0} \text { implies }\left\|H_{a}(x-a)\right\|<\varepsilon_{3}\left\|x_{0}-a\right\|
$$

$$
S\left(a, \delta_{0}\right)=\left\{x \in E \mid\|x-a\| \leqq \delta_{0}\right\} \subset \bar{G} .
$$

We can assume that $0 \notin \partial S\left(a, \delta_{0}\right)$. Since $H$ is completely continuous, the set $H\left[\partial S\left(a, \delta_{0}\right)\right]$ is bounded, namely,

$$
\|H(x)\| \leqq M \quad \text { for every } \quad x \in \partial S\left(a, \delta_{0}\right)
$$

for some $M>0$. Put $\varepsilon_{0}=\varepsilon_{2} \delta_{0} / 2 M$.

Now, assume that $|\varepsilon| \leqq \varepsilon_{0}$ and

$$
F\left(x_{0}\right)+\varepsilon H\left(x_{0}\right)=a x_{0}+(1-\alpha) a \text { for some } x_{0} \in \partial S\left(a, \delta_{0}\right) .
$$

Let us consider the mapping

$$
g(x)=f(x)-\varepsilon_{1} H(x) .
$$

This is uniformly increasing at $a$, and

$$
\begin{aligned}
g\left(x_{0}\right)-g(a) & =\left[f\left(x_{0}\right)-\varepsilon_{1} H\left(x_{0}\right)\right]-\left[f(a)-\varepsilon_{1} H(a)\right] \\
& =x_{0}-F\left(x_{0}\right)-\varepsilon_{1} H\left(x_{0}\right)+\varepsilon_{1} H(a) \\
& =x_{0}-\left[F\left(x_{0}\right)+\varepsilon H\left(x_{0}\right)\right]+\varepsilon H\left(x_{0}\right)+\varepsilon_{1}\left[H(a)-H\left(x_{0}\right)\right] \\
& =(1-\alpha)\left(x_{0}-a\right)+\varepsilon H\left(x_{0}\right)+\varepsilon_{1}\left[H(a)-H\left(x_{0}\right)\right],
\end{aligned}
$$

from which it follows that

$$
\begin{aligned}
\left\|g\left(x_{0}\right)-g(a)-(1-\alpha)\left(x_{0}-a\right)\right\| & \leqq|\varepsilon| \cdot\left\|H\left(x_{0}\right)\right\|+\varepsilon_{1}\left\|H(a)-H\left(x_{0}\right)\right\| \\
& \leqq|\varepsilon| \cdot M+\varepsilon_{1} \varepsilon_{3}\left\|a-x_{0}\right\| \leqq \varepsilon_{0} M+\frac{1}{2} \varepsilon_{2}\|a-x\| \\
& =\frac{1}{2} \varepsilon_{2}\left\|a-x_{0}\right\|+\frac{1}{2} \varepsilon_{2}\left\|a-x_{0}\right\|=\varepsilon_{2}\left\|a-x_{0}\right\| .
\end{aligned}
$$


Therefore, we have that $1-\alpha \geqq 0$, namely, $\alpha \leqq 1$, which, by the fixed point theorem mentioned above, implies that $F(x)+\varepsilon H(x)$ has a fixed point on $\overline{S\left(a, \delta_{0}\right)}$.

\section{References}

[1] Leray, J. and Schauder, J. Topologie et Equations Fonctionnelles, Ann. Sci. Ecole Norm. Sup., 51 (1934), $45 \rightarrow 78$.

[2] Nagumo, M., Degree of Mappings in Convex Linear Topological Spaces, Amer. J. Math., 73 (1951), 497-511.

[3] Yamamuro, S., A Note on the Boundedness Property of Non-linear Operators, Yokohama Math. J. 10 (1962), 19-23.

[4] Yamamuro, S., Some Fixed Point Theorems in Locally Convex Linear Spaces, ibid., $11(1963), 5-12$.

The Australian National University, Canberra. 